\title{
USP18 Gene
}

National Cancer Institute

\section{Source}

National Cancer Institute. USP18 Gene. NCI Thesaurus. Code C156940.

This gene plays a role in the cleavage of ubiquitin-like protein ISG15 conjug ates and the regulation of interferon-mediated signaling. 